\title{
Take a cold flower
}

\author{
Richard Amasino \\ Recent work shows that one pathway to flowering involves a complex that modulates the chromatin structure of the \\ flowering repressor FLOWERING LOCUS $\mathrm{C}$ and that a component of this flowering complex is also involved in \\ regulation of a cold-response gene.
}

Deciding when to flower is perhaps the most crucial developmental decision in the plant life cycle, and so flowering transition timing is regulated at many levels. In most plant species, flowering is influenced by environmental signals, such as day length and temperature, as well as by developmental state (e.g., size and age). Papers on pages 162-166 (ref. 1) and 167-171 (ref. 2) provide molecular insight into a single flowering pathway and provide a molecular connection between flowering and cold acclimation.

\section{The autonomous pathway}

The first studies of Arabidopsis thaliana flowering-time mutants were done in genetic backgrounds that flower rapidly ${ }^{3}$. In such backgrounds, mutants with delayed flowering are readily apparent (Fig. 1). These delayed-flowering mutants can be grouped into two classes: photoperiod and autonomous. A. thaliana flowering is promoted by long days. Photoperiod mutants are 'blind' to the promotive effects of long days, whereas autonomous-pathway mutants flower late in long days, like the photoperiod mutants, and even later in short days, thus retaining a photoperiod response. Their flowering is thus 'autonomous' of environmental cues (in this case, independent of day length) and is thought to be regulated in response to developmental cues ${ }^{3}$. This pathway has also been implicated in sensing differences in ambient growth temperature ${ }^{4}$. The molecular identities of five of the six loci that contribute to the autonomous pathway have been

Richard Amasino is in the Department of Biochemistry, University of Wisconsin, 433 Babcock Drive, Madison, Wisconsin 53706-

1544,USA.e-mail:amasino@biochem.wisc.edu described, all of which regulate flowering by repressing the expression of FLOWERING LOCUS C $(F L C)^{5}$, a flowering repressor. Autonomous-pathway mutants have elevated levels of FLC and delayed flowering.

\section{Regulation of flowering by chromatin modification}

FVE was the one remaining autonomouspathway gene that had not yet been identified. On page 162, Ausín et al. ${ }^{1}$ report that FVE

\section{a}
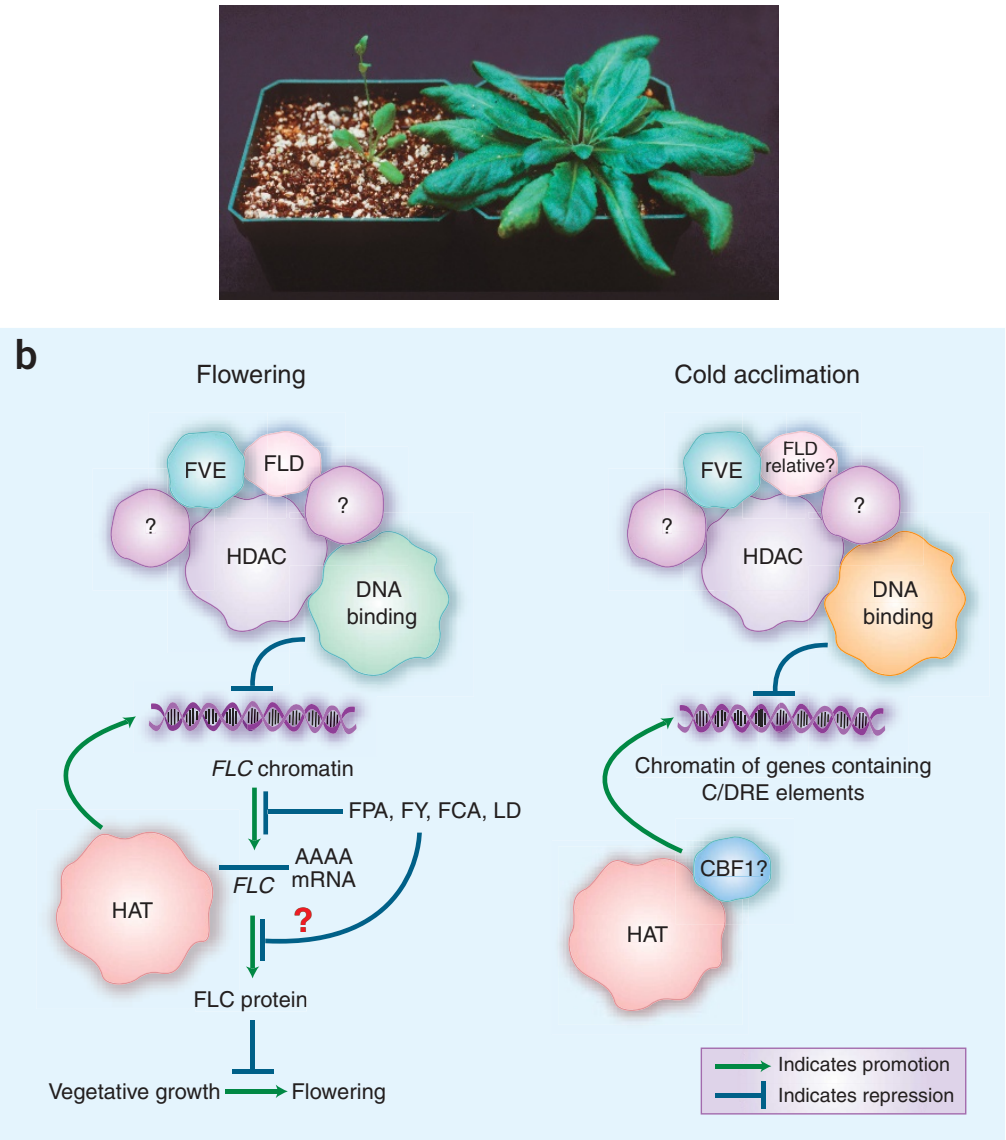

Figure 1 Regulation of expression of $F L C$ and cold-regulated genes.(a) Wild-type $A$. thaliana (left) and a delayed flowering mutant (right) grown under long-day conditions. Both plants are shown at the time of the flowering transition, and so the mutant is much older than the wild type. (b) A model for the regulation of expression of FLC and cold-regulated genes. HAT, histone acetyltransferase; HDAC, histone deacetylase. 
encodes a protein related to the mammalian retinoblastoma-associated proteins $\mathrm{RbAp} 46$ and $\mathrm{RbAp} 48$ and yeast multicopy suppressor of IRA1 (MSI). In yeast, fruit flies, mammals and maize, MSI-like proteins are present in a range of complexes involved in chromatin assembly or modification, including histone deacetylases (HDACs), histone acetyltransferase complexes (HATs), histone methyltransferases, chromatin assembly factor 1 (CAF-1) and the nucleosome remodeling factor $\mathrm{NURF}^{6-8}$. In A. thaliana, one of the MSI proteins (AtMSI1) is present in CAF-1 (ref. 9) as well as a complex similar to those of the Polycomb group in animals ${ }^{6}$.

FLC is a target of FVE, and fve mutants have high levels of FLC expression. Ausín et al. examined whether the state of FLC chromatin was altered in the fve mutant. Using antibodies against acetylated histones $\mathrm{H} 3$ or $\mathrm{H} 4$ in chromatin immunoprecipitation assays, they detected increased acetylation of FLC chromatin in the fve mutant, which probably causes the elevated FLC expression; it is well established in many systems that increased acetylation of $\mathrm{H} 3$ and $\mathrm{H} 4$ are associated with higher levels of gene expression ${ }^{10}$.

In studies of another autonomous-pathway gene, FLD, He et al. ${ }^{11}$ carried out chromatin immunoprecipitation assays with an antibody that preferentially recognized tetra-acetylated H4. Acetylation of $\mathrm{H} 4$ was greater in FLC chromatin of fld and fve mutants. These two studies indicate that FLD and FVE negatively regulate FLC by participating in the deacetylation of chromatin as part of an HDAC complex (Fig. 1). An unresolved issue is how the HDAC complex is recruited to FLC. Neither FVE nor FLD seem to bind DNA. In principle, mutations of the factors that bring HDACs to FLC should have delayed flowering like autonomous-pathway mutants. But other autonomous-pathway mutants do not have increased FLC acetylation. This indicates that other genes of the pathway probably encode proteins that regulate FLC by alternative mechanisms.

\section{Connection to cold signaling}

Kim et al. ${ }^{2}$ set out to study the acquisition of cold tolerance. When plants are exposed to cold temperatures, there is a rapid induction of genes that help to protect the plant from injury ${ }^{12}$. A DNA element (C/DRE) required for induction of several cold-induced genes had been identified previously ${ }^{12}$. Kim et al. identified a mutant, acg1, that had higher basal levels of a reporter gene containing multiple copies of the C/DRE element as well as higher levels of a cold-responsive gene, COR15a, that contains this element. acg1 mutants also had delayed flowering, and the mutation was identified as an allele of $f v e$.

Because FVE is involved in the deacetylation of $F L C$, it is possible that cold-responsive genes containing C/DRE elements are also subject to repression by deacetylation. It will be interesting to examine the acetylation state of the chromatin of such cold-responsive genes before and after cold induction. Another line of evidence that chromatin modification may be involved in cold-induced gene expression is the observation that a cold-induced factor that binds to the C/DRE element, CBF1, can associate with proteins that have HAT activity ${ }^{12}$. Thus, the cold induction of genes containing C/DRE elements may result from coldincreased HAT activity that counteracts HDAC-mediated repression (Fig. 1). In the absence of HDAC-mediated repression (e.g., in fve mutants), a higher basal level of expression of genes containing C/DRE elements would be expected, as well as faster cold induction of such genes, as observed by Kim et al.

Kim et al. also found that the flowering of wild-type plants, but not fve mutants, is developmentally delayed when they are subjected to intermittent cold. The authors suggest that FVE has a dual role in regulating flowering time and cold acclimation, and that this may be an adaptation to the fluctuating temperatures of spring. It will be interesting to test this suggestion by determining whether other autonomous-pathway mutants have delayed flowering when exposed to intermittent cold.
If FVE does in fact regulate genes containing C/DRE elements by histone deacetylation, and if, as Kim et al. suggest, FVE has a direct role in cold regulation, then cold would be expected to reduce FVE protein levels or activity. Alternatively, FVE may be part of a complex that provides constitutive repression, and cold may induce other factors (such as CBFs) that recruit competing chromatinmodeling complexes (such as HATs) to genes containing C/DRE elements.

In contrast to fve mutants, expression of C/DRE element-containing genes like COR15a is not detectably elevated in fld (I. Lee, personal communication). This indicates that FVE could function in an HDAC complex with FLD to control flowering time, and in a complex without FLD to regulate cold-responsive genes. Because FLD and FLC are preferentially expressed in shoot and root apical regions ${ }^{11}$, whereas cold acclimation occurs throughout the plant, FVE probably has some different partners in the regulation of FLC versus coldresponsive genes. There are two FLD relatives in the A. thaliana genome ${ }^{11}$, and perhaps one or both of these join FVE in regulating coldresponsive genes. FVE might also affect expression of cold-responsive genes in a different type of HDAC complex or, as MSI-like proteins are found in a wide range of chromatin-modifying complexes, FVE could regulate cold-responsive genes independently of HDAC activity.

1. Ausín, I., Alonso-Blanco, C., Jarillo, J.A., Ruiz-García, L. \& Martínez-Zapater, J.M. Nat. Genet. 36, 162-166 (2004).

2. Kim, H.-J. et al. Nat. Genet. 36, 167-171 2004).

3. Simpson, G.G. \& Dean, C. Science 296, 285-289 (2002).

4. Blasquez, M.A., Ahn, J.H. \& Weigel, D. Nat. Genet. 33, 168-171 (2003).

5. Michaels, S.D. \& Amasino, R.M. Plant Cell 13, 935-941 (2001).

6. Köhler, C. et al. EMBO J. 22, 4804-4814 (2003).

7. Nicolas, E. et al. J. Biol. Chem. 275, 9797-9804 (2000).

8. Rossi, V. et al. Plant Mol. Biol. 51, 401-413 (2003). 9. Kaya, H. et al. Cell 104, 131-142 (2001).

10. Carrozza, M.J., Utley R.T., Workman, J.L. \& Cote, J. Trends Genet. 19, 321-329 (2003).

11. He, Y., Michaels, S.D. \& Amasino, R.M. Science 302, 1751 (2003)

12. Vlachonasios, K.E., Thomashow, M.F. \& Triezenberg, S.J. Plant Cell 15, 626-638 (2003). 